\title{
Osteopathic Treatment Approach to Psychoemotional Trauma by Means of Bifocal Integration
}

Torsten Liem, MSc Ost, MSc Paed Ost, DO (Germany); Prof Winfried Neuhuber, MD

From the Osteopathie Schule Deutschland in Hamburg, Germany (Mr Liem) and the

Institut für Anatomie at Friedrich-Alexander-University Erlangen-Nürnberg (Prof Neuhuber) in Erlangen, Germany.

Financial Disclosures: None reported.

Support: None reported. Address correspondence to Torsten Liem, MSc Ost, MSc Paed Ost, Osteopathie Schule Deutschland, Mexikoring 19, 22297 Hamburg, Germany.

Email: tliem@osteopathieschule.de Submitted December 17, 2018; revision received February 19, 2019; accepted March 1, 2019.
Traumatic psychoemotional experiences often manifest in hypersympathetic states of excitement or in immobilization and withdrawal behaviors. A person with this kind of traumatic background may present with an autonomic response consistently maintained in the defensive state, which over time becomes a stressor. In this article, the authors discuss an osteopathic approach to psychoemotional trauma by means of bifocal integration, with an emphasis on promoting a relaxed body and mind by actively involving the patient in the healing process. They briefly discuss the role of mind-body therapies in treatment, considering the popular polyvagal theory and other neuropsychophysiological frameworks. The authors also focus on clinical practice, introducing the reader to the different elements of the osteopathic treatment, including the acknowledgement and integration of top-down and bottom-up dynamics in diagnosis and therapy.

J Am Osteopath Assoc. 2020;120(3):180-189 doi:10.7556/jaoa.2020.021

Keywords: emotional integration, mind-body therapy, psychoemotional trauma, trauma

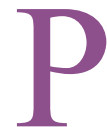
sychoemotional trauma is generally understood to refer to a highly stressful mental or physical event. Trauma can be defined as an "inescapably stressful event that overwhelms people's existing coping mechanisms."1 This definition of trauma includes responses to single incidents, such as natural disasters, accidents, deaths, and crimes, as well as to chronic experiences such as childhood abuse, neglect, violent relationships, and deprivation. ${ }^{2}$ It is important to understand that the traumatic event is defined by the experience of the survivor; 2 people could experience the same event and one of them may perceive this experience as traumatic, whereas the other may not. ${ }^{3}$

The effects of traumatic experiences can be manifold. For example, trauma survivors may experience anxiety and depression, substance abuse, eating or personality disorders, or posttraumatic stress disorder (PTSD). ${ }^{2}$ Traumatized children and adults lose their capacity to use emotions for effective actions, so they may fail to correctly identify their own sensations, emotions, and physical states, which can extend into difficulty appreciating emotional states and needs of others. ${ }^{4}$

On a physiological level, trauma stems from a failure of the physiological activation and hormonal response to organize effective behavior toward a threat. While in a healthy individual, a successful fight or flight response would be produced, the traumatized individual often becomes immobilized, resulting in a conditioned behavioral response. ${ }^{4}$ 
Complementary therapy approaches - in particular, mind-body therapies (MBTs) - have received increasing interest, as they are perceived to decrease trauma-related symptoms and emotional dysregulation. ${ }^{5,6}$ Mind-body therapies place an emphasis on the interaction between the brain and the body. They are practiced in a way to facilitate the effect of the mind on the body and typically include psychological, behavioral, spiritual, and social approaches, such as cognitive behavioral therapy (CBT), meditation, breathwork, and imagery.

Previous research has shown that patients with PTSD using MBTs reported positive effects on their PTSD symptoms, including therapeutic effects on stress responses; reductions in anxiety, depression, and anger; an increase in pain tolerance; improved selfesteem; and an enhanced ability to relax and to cope with stressful situations. ${ }^{6}$ Specifically, trauma-focused CBT has been recommended for PTSD by various treatment and expert consensus guidelines, ${ }^{7,8}$ highlighting the effectiveness of CBT for PTSD in adults who have experienced a range of traumatic events. ${ }^{9,10}$

By focusing on a combination of somatic awareness in the form of interoception, proprioception, and mindfulness, MBTs allow for nonjudgement and acceptance. Patients are supported and guided in a process of reorientation in order to promote adaptability and resilience to perceived stressors. ${ }^{11,12}$ Mind-body therapies are an effective tool for the regulation of vagal function, including the facilitation of self-regulatory skills and resilience of the autonomic nervous system. ${ }^{13-16}$

In this article, we describe an osteopathic approach to psychoemotional trauma by means of emotional integration in the context of available research findings, and we include a short case report of a patient to highlight the different elements of this treatment approach in clinical practice.

\section{The Polyvagal Theory}

In the years since it was first described by Stephen Porges in $1995,{ }^{17,18}$ the polyvagal theory (PVT) has garnered interest from MBT therapists worldwide, particularly with respect to the treatment of patients with trauma. The PVT is an attempt to explain the relationship between parasympathetic activity and behavior from an evolutionary perspective, and it aims to provide a framework for understanding the connections between the processes of the brain and body. ${ }^{17,18}$

The term polyvagal refers to 2 vagal platforms. First is the phylogenetically "older" unmyelinated system represented by the dorsal motor nucleus (DMX), which innervates subdiaphragmatic organs (in particular, the gastrointestinal tract) and is associated with immobilization and dissociation. The second platform is the "newer" system, which is represented by the nucleus ambiguus (NA) in the ventrolateral medulla; it contains myelinated nerve fibers, is associated with safety and social behavior, and regulates the heart and lungs to facilitate states of calmness. ${ }^{19}$ The myelinated vagus nerve is said to occur only in mammals. ${ }^{19}$

However, evidence from comparative anatomical and functional studies argues against this proposed phylogenetic rooting of PVT. Although the NA is the source of myelinated cardioinhibitory axons in mammals, the cardioinhibitory vagal neurons are myelinated, conducting at velocities between 7 and $35 \mathrm{~ms}$ (which is equivalent to mammalian B fibers) as early as in elasmobranchs dating back 400 million years, and they have their cell bodies at 2 different locations in the medulla (the DMX and the primordium of the NA). ${ }^{20,21}$ Also, lungfish, which are positioned at the evolutionary base of air-breathing species, have the myelinated cardiac vagus nerve originating in dorsal and ventrolateral brainstem nuclei. ${ }^{22}$ These myelinated, fast-conducting axons enable the beat-to-beat slowing of the heart rate that is mandatory for cardiorespiratory interactions observed in these old vertebrates, similar to the respiratory sinus arrhythmia of mammals. ${ }^{22,23}$ The unmyelinated cardiac neurons of the DMX do not have any significant influence on heart rate and thus cannot be responsible for bradycardia such as that observed in freezing states. They appear to influence ventricular inotropy and may protect the cardiomyocytes against ischemia. ${ }^{24}$ 
The PVT outlines 3 distinct categories of response to perceived risk: feeling safe, in danger, or a threat to life. These categories are phylogenetically arranged and are associated with the adaptive behaviors of social communication (facial expression, vocalization, listening) controlled by the NA, as well as the defensive mobilization (combat, evacuation), and defensive stagnation (vasovagal syncopes, dissociation, freeze behavior) controlled by the DMX. ${ }^{17,19,24-26}$

Again, the proposed link of these behavioral phenomena to the old unmyelinated and new myelinated vagus nerve is misleading. The NA does not control facial expression because the mimic muscles are innervated by the facial nerve, nor does it control the middle ear muscles (tensor tympani, innervated by the motor branch of the trigeminal nerve, and stapedius, innervated by the facial nerve) or other cranial and neck muscles as suggested by the $\mathrm{PVT}^{17}$ except, of course, those of pharynx and larynx.

Although both the motor nucleus of the trigeminal and the facial nucleus belong - together with the NA and the spinal nucleus of the accessory nerve, which innervates the sternocleidomastoid and trapezius muscles - to the special visceral efferent column (motoneuronal nuclei of cranial nerves V, VII, IX, X, and XI supplying muscles derived from the pharyngeal or branchial arches, thus also called branchial motor), ${ }^{27}$ they are not coordinated by the new vagus nerve, but rather by premotor networks in the lateral parvicellular and intermediate reticular formation. ${ }^{28-32}$ The intermediate reticular formation, located between the medial magnocellular and lateral parvicellular area, harbors the neuronal networks for cardiovascular regulation and the central pattern generators for breathing, swallowing, and vomiting. The DMX and NA are embedded in these networks. Vagal afferents are connected via the nucleus of the solitary tract not only to the motor vagus nuclei (DMX and NA), but also to the premotor networks of the reticular formation. ${ }^{32}$ Equally important are trigeminal and upper cervical spinal afferents, which also feed into the premotor reticular networks.
Behavioral states such as fight and flight, immobilization, freezing, and risk assessment—with their concomitant motor, autonomic, and endocrine effectsare orchestrated by the mesencephalic periaqueductal gray (PAG). ${ }^{33-36}$ The PAG is reciprocally connected, on one hand, to the hypothalamus and the limbic system (in particular the amygdala and the prefrontal cortex $)^{34,35}$ and, on the other hand, to various premotor and autonomic brainstem nuclei coordinating respiration and the emotional motor system. ${ }^{36}$ The PAG receives afferents from almost all sensory systems (not least of which is the nociceptive system) and modulates their processing. ${ }^{35}$

Nevertheless, the vagus nerve has a significant impact on emotions and other behavioral states by virtue of its large afferent portion. Vagal afferents, which account for about $80 \%$ of vagus axons, are relayed through the nucleus of the solitary tract to the PAG, hypothalamus, amygdala, as well as the insular, cingulate, and prefrontal cortices, where they are integrated into emotional and cognitive processes. ${ }^{37-40}$ Recent studies have indicated that subdiaphragmatic vagal afferents influence innate anxiety, learned fear, and other behaviors. ${ }^{41,42}$ Furthermore, vagal afferents profoundly modulate spinal nociceptive processing in various experimental models. ${ }^{43,44}$

\section{Mechanisms of MBT}

Top-down therapies, such as clinical hypnosis, imagery, or meditation, initiate conscious and intentional mental processes at the level of the cerebral cortex. ${ }^{45}$ They cause a shift in cerebral function involving reorganization of neural representations within the central nervous system and enhanced bidirectional communication between the cerebral cortex and the limbic and brainstem structures that regulate autonomic, neuroendocrine, and emotional behaviors. ${ }^{37-39}$ In contrast, bottom-up therapies (eg, progressive muscle relaxation) work by stimulation of somatic, visceral, and/or chemosensory receptors, influencing central neural processing and mental processes via ascending 
pathways from the periphery to the cerebral cortex, resulting in increased heart rate variability and reduced expression of cytokines. ${ }^{37,40,47,48}$

Taylor et $\mathrm{al}^{45}$ proposed an integrative psychophysiological framework to illustrate the underlying mechanisms of MBTs, recognizing the multiple levels of the neuraxis at which mind-body interactions occur. According to this model, symptoms of stress manifest as functional disturbances of the executive homeostatic network, ie, imbalances in prefrontal cortex activation and higher-order visceral manifestations encoded on the prefrontal cortex, insular cortex, and anterior cingulate cortex. These disturbances are subsequently expressed at the periphery as reduced heart rate variability and expression of proinflammatory cytokines. ${ }^{45}$ Taylor et $\mathrm{al}^{45}$ suggest that effective MBTs should induce functional changes aimed at regional executive homeostatic network activation or increased heart rate variability and reduced cytokine production. ${ }^{45}$

\section{Neuroception of Safety in the Therapeutic Relationship}

Previous research has shown that the therapeutic relationship is paramount in a desired therapeutic outcome and that therapeutic outcomes may only minimally depend on the use of specific techniques. ${ }^{48-50}$

Effective therapeutic outcomes are only possible when patients feel safe in the therapy environment. Feelings of safety for the patient and the development of a healthy therapeutic relationship often depends on the therapist's ability to be fully present and engaged. ${ }^{51,52}$ Geller and Porges ${ }^{53}$ proposed that this relationship requires the therapist to be grounded, centered, and steady, as well as open and receptive to the patient's experience. The authors presented a new perspective on how specific features of therapeutic presence can improve a patient's the sense of safety. For example, it would be therapeutically beneficial for therapists to communicate with their clients using nonverbal markers, such as a warm voice, soft eye contact, open body posture, and a receptive stance. Through a consistent presence, the therapist can regulate the stress responses of the client; this approach can lead to a synergistic relationship wherein a patient's reactive capacity can be transformed over time into nondefensive social engagement. ${ }^{53}$ The therapist should be attuned to the patient's experience and physiology, responding in a way that will encourage a patient to feel open and present. $^{53}$ Additionally, patients can benefit from neural exercises that promote the experience of inner safety, such as deep abdominal breathing, social play, yoga, meditation, mindfulness, and being in nature. ${ }^{53}$

\section{Emotional and Bifocal Integration}

Traumatic experiences often manifest in hypersympathetic states of excitement or in immobilization and withdrawal behaviors. As time goes on, these initially life-saving responses become stressors. A person with a traumatic background may present with an autonomic response consistently maintained in the defensive state, which inhibits the downregulation of the defensive system.

Osteopaths (foreign-trained practitioners of osteopathy) enable patients to perceive and integrate physical reaction patterns by conveying to their patients an environment of trust and safety. The aim of osteopathic manipulative therapy (manipulative care provided by foreign-trained osteopaths) is to promote a relaxed body and mind, actively blocking hyperexcitability and sympathetic effects on the heart, as well as attenuating the hypothalamic-pituitary-adrenal axis. With the support of osteopathic techniques developed by one of the authors (T.L.) that facilitate relaxation, such as osteopathic heart-focused palpation (Figure 1) or osteopathic felt-sense (a process by which the osteopath palpates regions of greatest rhythmic flow and vitality while the patient focuses on sensations related to the palpated regions), ${ }^{54}$ past traumatic experiences of the patient (with further trauma confrontation during the course of the treatment) can be increasingly associated with relaxed body feedback, including calm breathing and heart rate, as well as relaxed facial muscles. This state is achieved and reinforced by vagal 


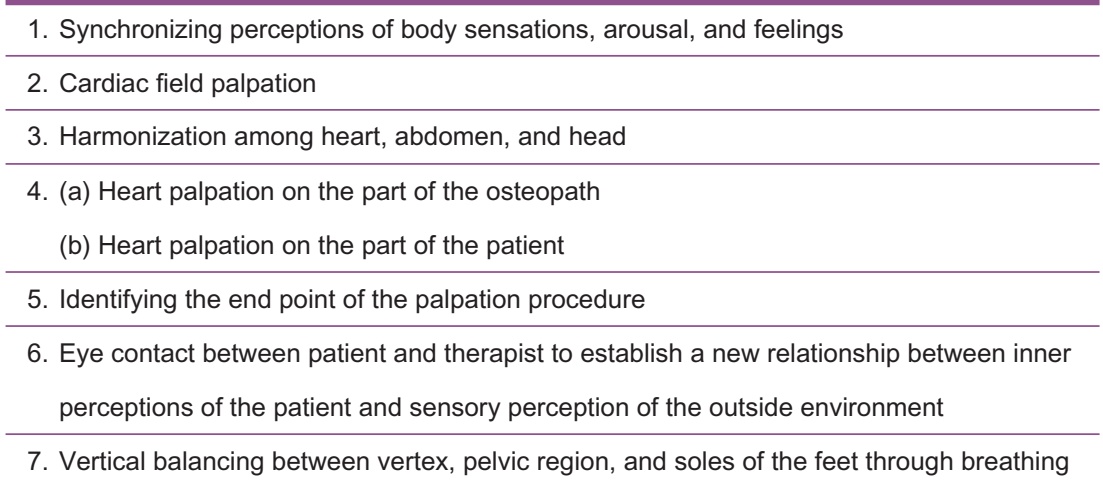

Figure 1.

Osteopathic heart-focused palpation technique for the treatment of patients with psychological trauma. ${ }^{54}$

cardiopulmonary and trigeminal afferent nerves (from sensors in the myofasciocutaneous system of the face), which activate PAG-limbic-prefrontal circuits.

In a resource-rich state, an awareness of the patient's life circumstances and past experiences, current behaviors, and inner feelings are combined with an acknowledgment of related disorders and dysfunctions. The patient is actively involved in the healing process, with the treatment helping the patient to acquire the competencies to progressively access this relaxed state in everyday life. Additionally, patients are encouraged to direct their attention to the present rather than the past, to recognize and acknowledge boundaries, to establish positive and fulfilling relationships with themselves and others, and to follow goals that give meaning to their life. ${ }^{46}$ Patients most likely will feel safe and sufficiently protected when a calm practice atmosphere is provided, including an empathetic therapist with good communication skills, a relaxed palpation style, and treatment sessions without time pressure. ${ }^{55}$ Additionally, as Elkiss and Jerome ${ }^{56}$ highlighted, the relationship between the therapist who touches and the patient who is being touched offers a powerful and intimate liaison that complements the equally empathetic communication of words and thus facilitates synchronized therapeutic healing.

The basis of this proposed treatment approach is the acknowledgment and integration of top-down and bottom-up dynamics in diagnosis and therapy (Figure 2).
One of the authors (T.L.) uses a system of emotional integration when treating patients with trauma, which includes a specific palpation practice that enables the patient to understand the relationship between impaired function, somatic dysfunction, and internal and external factors. ${ }^{55}$ The tissue palpation takes into consideration human developmental dynamics as well as breathing techniques derived from yoga, resource work, positive psychology, bilateral stimulation, vipassana meditation and body therapy, neurolinguistic body programming, neurogenic hesitation, somatic experiencing, nonviolent communication, and meditation.

The treatment is structured in 4 phases:

1. Establishment of the therapeutic relationship

2. Stabilization phase

3. Confrontation phase with bifocal integration

4. Integration into everyday life

During establishment of the therapeutic relationship, the goals of the treatment are discussed and agreed upon, and a transparent and stable therapeutic relationship is developed. The patient's needs (eg, for staying in control) and coping strategies are acknowledged by the patient as well as by the therapist. ${ }^{57}$ Deceleration of the therapeutic process is crucial at this stage. The therapist will confirm the presence of emotional centers in the body through palpation of tensions around the solar 


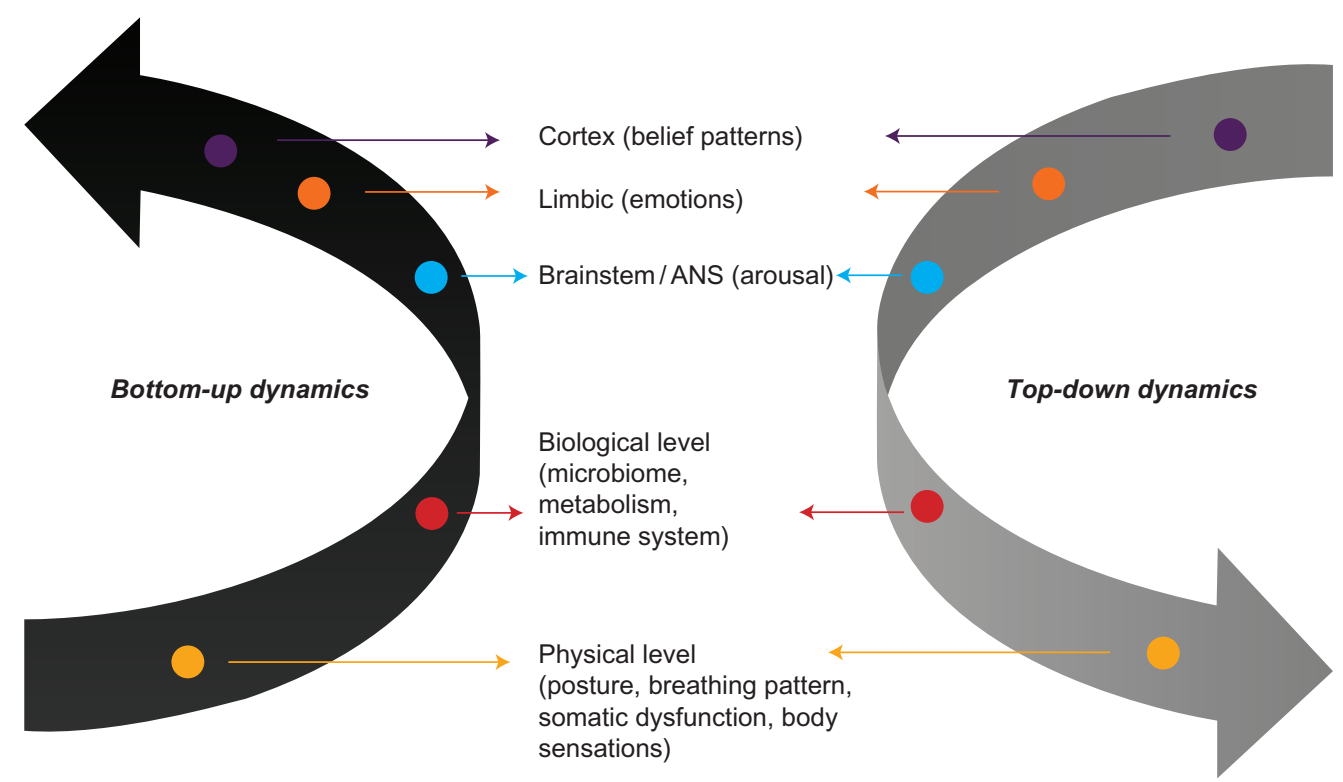

Figure 2.

Top-down and bottom-up process integration into the therapeutic encounter. In the context of dysfunctional influences as well as in the process of recovery, a range of ascending and descending dynamics interact as top-down and bottom-up mechanisms between peripheral tissues and the brain, including the immune systems and the nervous systems, which contribute to both physical and mental health. For example, the belief pattern of "life is uncertain" may trigger emotions, such as fear, in the limbic system, which may cause heightened sympathetic activity with increased, shallow breathing and body sensations of tension headaches, damp and cool hands and tensions in the neck and shoulder regions. Abbreviation: ANS, autonomic nervous system.

plexus/coeliacus, the suboccipital regions, and neck, eye, and heart regions, and will examine transition areas in the spine as well as other signs of traumatic stress. Palpation involves, for example, the coeliac ganglion, other sympathetic ganglia, the heart region, throat, suboccipital region, eyes, periphery and central sensitisation, pressure gradients, and reflex testing.

During the stabilization phase, stress-reducing concepts are used primarily, including identifying coping strategies, gaining knowledge about the consequences of trauma, exercising to reduce stress, differentiating and regulating emotions/affects, and developing trust. This phase comprises psychoeducation and deep learning, including the consideration of additional stress factors that may contribute to the current state-psychological stress, social factors, certain foods and xenobiotics, infections, nicotine, alcohol and drug abuse, oxidative stress, electrosmog, microbiome of the gut, and a sedentary life- style. The methods used during this phase strengthen patients' emotions for potential confrontational approaches to distressing topics during later phases of trauma confrontation and treatment. It also involves functional osteopathic manipulative therapy of the body regions that manifest the effects of psychological trauma.

The confrontation phase, specifically by means of bifocal integration, aims to work through the traumatic situation in a clearly defined setting. It includes anchoring and awareness of interoceptions, body sensations, arousals, emotions, thoughts, and pictures.

A simplified description of bifocal integration is as follows:

1. Anchoring of resources: The patient engages in anchoring of resources, eg, establishing a safe place.

2. Identifying the most arousing situation/picture: The patient identifies the most stressful situation/ 
picture related to the stress/trauma and scales it from 0 to 10 .

3. Associated body region: The patient perceives the region in the body that reacts the strongest and even increases the level of stress-related arousal.

4. Line of sight: The patient identifies the eye position/viewing direction that increases the level of arousal.

5. Pleasant body region: The patient identifies the region of the body that feels the most pleasant.

6. Line of sight: The patient identifies the eye position/viewing direction that increases the wellbeing and pleasant feeling in the previously identified body region.

7. Further resources: If necessary, more resources are anchored, such as archetypes, eg, inner healer; belief patterns; music; breathing eg, reduce the breathing rate by half.

8. Perception of arousal: The therapist acts as coregulator, identifying the level of stress-related arousal during each moment of the process.

9. Bifocal shifting of the focus: The patient dynamically shifts the focus between the 2 identified viewing directions in his or her own time.

10. Palpation: At the same time, the therapist palpates the identified body region, which increases the arousal and/or the most pleasant body region and associated patterns of tension and resources depending on the reactions that occur in the patient. Further specific palpatory awareness approaches are applied.

11. Coregulation: If a slight arousal is perceived, the process continues with its own speed and flow, with the lowest amount of intervention by the therapist. In the event of a stronger stress-related arousal, the patient is asked to breathe more slowly. In the event of entering a freeze state, the patient is asked to breathe more deeply.

12. Increase of arousal: The patient tries to increase any arousal related to the situation.

13. Anchoring of resources: The attention of the patient is guided through different regions of the body by an instructor to encourage the experience of specific body sensations.

During the process, the patient keeps in touch with his or her resources and with the details of his or her trauma while the therapist ensures that the patient, who is usually in a slightly sympathetic arousal state, is at the same time anchored in a relaxed state. ${ }^{57}$ Slight overexcitation of the sympathetic nervous system can occur if the patient is mentally brought back into the stressful traumatic situation. However, the osteopathic intervention should aim to prevent overexcitation of the sympathetic nervous system due to stimulation of PAG-limbic circuits. This process is characterized by transformative dynamics, and the main question to be answered is not "What is it?" but rather "What happens?"58,59 To accompany the inner experience of the treatment, tissue palpation is essential. Palpation and its energetic interactions in the body fields are combined with respiration, neurogenic hesitation, meditation, dual awareness, and inner dialogue. Patients perceive their interoception in a differentiated manner, and implicit memories become explicit. ${ }^{55}$ A significant step for patients is the integration of their progress into everyday life. During the treatment, essential qualities for this integration are promoted on an ongoing basis, such as being present, self-aware, and open to life. ${ }^{60}$ Ultimately, by being able to be centered in the state of attentive motor and autonomic relaxation, patients are increasingly able to experience liveliness, interconnectedness, and an alert presence that no longer supports dysfunctional patterns and self-contraction. ${ }^{59}$

\section{Clinical Case Description}

A 42-year-old woman presented with low back pain, which she had for many years. Her medical history revealed evidence of prenatal stress, and physical abuse in childhood was mentioned. She tested positive for adverse childhood experiences (5/10 points).

Specific events in her childhood-particularly between the ages of 5 and 7 years, during which she was physically abused-were still very burdensome 
for her $(9.5 / 10$ points on a numerical rating scale assessing stress levels, with 0 indicating no stress at all and 10 meaning the worst stress imaginable).

A complete osteopathic structural examination was performed, which consisted of palpation to assess muscles, ligaments, and tendons, looking for asymmetries and tenderness. ${ }^{61}$ After ensuring that enough resources were available, the active part of bifocal integration was initiated. Adapted from the recently developed brainspotting method, ${ }^{62}$ the patient envisioned the most distressing moment of the specific traumatic situation and localized the body region most uncomfortably responsive to it - in this case, the solar plexus, which felt like a contracted fist. The eye position that intensified this unpleasant physical sensation was localized by asking the patient to slowly let her eyes wander horizontally and then vertically and by looking in the near or far line of vision to find the line of vision that increased stress.

Subsequently, the body region that felt most comfortable was located. This was the sternum region, which on palpation felt strong. Subsequently, the eye position was localized, which enhanced this pleasant body sensation (resource-enhancing line of vision).

The patient was asked to rhythmically move her eyes back and forth from the resourceful and stress-enhancing eye positions. The therapist palpated the sternum region and followed in micromovements the course of the patient's eyes. After that, the therapist palpated the region of the solar plexus and also followed the eyes of the patient in micromovements.

At the same time, all vegetative signs of the patient were observed (eg, breathing), and the patient was asked to remain open for whatever happened. Over the next 30 minutes, the subjective burden decreased from 9.5 to 0.5 on the stress scale. The tension in the solar plexus also decreased. Breathing, which accelerated during the treatment session, deepened and slowed down. In the subsequent treatment, 3 weeks later, the perceived burden at the beginning of the session had increased again to $6 / 10$ on the stress scale; however, during the course of 2 further treatments, the perceived burden remained permanently at 0 . Further interventions were provided, as well as stress-reducing and affect-differentiating and regulating exercises performed by the patient between the sessions.

\section{Conclusion}

This article provides a short introduction to the neuropsychophysiologic framework of psychoemotional trauma and how it is used to explain the effects of MBTs and effective patient-therapist interactions. Establishment of trust and safety for the patient is the first and paramount step, followed by a stabilization phase that includes stress-reducing exercises and the integration of coping strategies. The confrontation phase works through the traumatic event in a defined setting, including anchoring and inner dual awareness of interoceptions. Finally, the patient is encouraged to integrate the newly built emotional and social confidence into everyday life.

\section{References}

1. Van der Kolk BA, Fissler R. Dissociation and the fragmentary nature of traumatic memories: overview and exploratory study. J Trauma Stress. 1995;8(4):505-525. doi:10.1007/bf02102887

2. Giller E. What is psychological trauma? https://www.sidran.org/ resources/for-survivors-and-loved-ones/what-is-psychological-trauma/. Accessed December 15, 2018.

3. Allen JG. Coping With Trauma: A Guide to Self-Understanding. Washington, DC: American Psychiatric Press; 1995.

4. Van der Kolk BA. Clinical implications of neuroscience research in PTSD. Ann N Y Acad Sci. 2006;1071(1):277-293. doi:10.1196/ annals. 1364.022

5. Wynn GH. Complementary and alternative medicine approaches in the treatment of PTSD. Curr Psychiatry Rep. 2015;17(8):600. doi:10.1007/ s11920-015-0600-2

6. Kim SH, Schneider SM, Kravitz L, Mermier C, Burge MR. Mind-body practices for posttraumatic stress disorder. J Investig Med. 2013;61 (5):827-834. doi:10.2310/JIM.0b013e3182906862

7. Post-traumatic stress disorder. National Institute for Health and Care Excellence. https://www.nice.org.uk/guidance/ng116/chapter/ Recommendations\#management-of-ptsd-in-children-young-peopleand-adults. Accessed February 1, 2019.

8. American Psychological Association. Clinical Practice Guideline for the Treatment of PTSD. Washington, DC: American Psychological Association; 2017. https://www.apa.org/ptsd-guideline/ptsd.pdf. Accessed February 1, 2019. 
9. Kar N. Cognitive behavioral therapy for the treatment of post-traumatic stress disorder: a review. Neuropsychiatr Dis Treat. 2011;7:167-181. doi:10.2147/NDT.S10389

10. Cusack K, Jonas DE, Forneris CA, et al. Psychological treatments for adults with posttraumatic stress disorder: a systematic review and meta-analysis. Clin Psychol Rev. 2016;1(43):128-141. doi:10.1016/j. cpr.2015.10.003

11. Mehling WE, Wrubel J, Daubenmier JJ, et al. Body awareness: a phenomenological inquiry into the common ground of mind-body therapies. Philos Ethics Humanit Med. 2011;6(1):1. doi:10.1186/ 1747-5341-6-6

12. Farb N, Daubenmier J, Price CJ, et al. Interoception, contemplative practice, and health. Front Psychol. 2015;6:763. doi:10.3389/ fpsyg.2015.00763

13. Porges SW and Carter CS. Polyvagal theory and the social engagement system: neurophysiological bridge between connectedness and health. In: Gerbarg PL, Muskin PR, Brown RP, eds. Complementary and Integrative Treatments in Psychiatric Practice. Arlington, VA: American Psychiatric Association Publishing; 2017:221-240

14. SchmalzI L, Powers C, Henje Blom E. Neurophysiological and neurocognitive mechanisms underlying the effects of yoga-based practices: towards a comprehensive theoretical framework. Front Hum Neurosci. 2015;9:235. doi:10.3389/ fnhum.2015.00235

15. Streeter CC, Gerbarg PL, Saper RB, Ciraulo DA, Brown RP. Effects of yoga on the autonomic nervous system, gamma-aminobutyric-acid, and allostasis in epilepsy, depression, and post-traumatic stress disorder. Med Hypotheses. 2012;78(5):571-579. doi:10.1016/j. mehy.2012.01.021

16. Muehsam D, Lutgendorf S, Mills PJ, et al. The embodied mind: a review on functional genomic and neurological correlates of mind-body therapies. Neurosci Biobehav Rev. 2017;73:165-181. doi:10.1016/j. neubiorev.2016.12.027

17. Porges SW. The polyvagal theory: phylogenetic substrates of a social nervous system. Int J Psychophysiol. 2001;42(2):123-146. doi:10.1016/s0167-8760(01)00162-3

18. Porges SW. The polyvagal theory: phylogenetic contributions to social behavior. Physiol Behav. 2003;79(3):503-513. doi:10.1016/ s0031-9384(03)00156-2

19. Porges SW. The Polyvagal Theory: Neurophysiological Foundations of Emotions, Attachment, Communication, and Self-Regulation. New York, NY: Norton; 2011.

20. Barrett DJ, Taylor EW. The location of cardiac vagal preganglionic neurones in the brain stem of the dogfish Scyliorhinus canicula. J Exp Biol. 1985;117:449-458.

21. Barrett DJ, Taylor EW. The characteristics of cardiac vagal preganglionic motoneurones in the dogfish. $J$ Exp Biol. 1985;117:459-470.

22. Monteiro DA, Taylor EW, Sartori MR, Cruz AL, Rantin FT, Leite CA. Cardiorespiratory interactions previously identified as mammalian are present in the primitive lungfish. Sci Adv. 2018;4(2):eaaq0800. doi: $10.1126 /$ sciadv.aaq0800

23. Grossman P, Taylor EW. Toward understanding respiratory sinus arrhythmia: relations to cardiac vagal tone, evolution and biobehavioral functions. Biol Psychol. 2007;74:263-285. doi:10.1016/j. biopsycho.2005.11.014
24. Porges SW. Love: an emergent property of the mammalian autonomic nervous system. Psychoneuroendocrinology. 1998;23:837-861.

25. Porges SW. The polyvagal perspective. Biol Psychol 2007;74:116-143. doi:10.1016/j.biopsycho.2006.06.009

26. Porges SW. The polyvagal theory: new insights into adaptive reactions of the autonomic nervous system. Cleve Clin J Med. 2009;76(suppl 2: S86-S90. doi:10.3949/ccjm.76.s2.17

27. Fritzsch B, Elliott KL, Glover JC. Gaskell revisited: new insights into spinal autonomics necessitate a revised motor neuron nomenclature. Cell Tissue Res. 2017;370:195-209. doi:10.1007/ s00441-017-2676-y

28. Fay RA, Norgren R. Identification of rat brainstem multisynaptic connections to the oral motor nuclei using pseudorabies virus: I: masticatory muscle motor systems. Brain Res Brain Res Rev. 1997;25 (3):255-275. doi:10.1016/s0165-0173(97)00026-x

29. Fay RA, Norgren R. Identification of rat brainstem multisynaptic connections to the oral motor nuclei in the rat using pseudorabies virus: II: facial muscle motor systems. Brain Res Brain Res Rev. 1997;25(3):276-290. doi:10.1016/s0165-0173(97)00027-1

30. Fay RA, Norgren R. Identification of rat brainstem multisynaptic connections to the oral motor nuclei using pseudorabies virus: III: lingual muscle motor systems. Brain Res Brain Res Rev. 1997;25 (3):291-311. doi:10.1016/s0165-0173(97)00028-3

31. Travers JB, Norgren R. Afferent projections to the oral motor nuclei in the rat. J Comp Neurol. 1983;220(3):280-298. doi:10.1002/ cne. 902200303

32. Solitary nucleus (SOL). In: Büttner-Ennever JA, Horn AKE, eds Olszewski and Baxter's Cytoarchitecture of the Human Brainstem, 3rd ed. Basel: Karger; 2014.

33. Deng $\mathrm{H}$, Xiao $\mathrm{X}$, Wang Z. Periaqueductal gray neuronal activities underlie different aspects of defensive behaviors. J Neurosci. 2016;36 (29):7580-7588. doi:10.1523/JNEUROSCI.4425-15.2016

34. Roelofs K. Freeze for action: neurobiological mechanisms in animal and human freezing. Philos Trans R Soc Lond B Biol Sci. 2017;372 (1718):20160206. doi:10.1098/rstb.2016.0206

35. Jänig W. The Integrative Action of the Autonomic Nervous System. Cambridge, UK: Cambridge University Press; 2006.

36. Holstege $\mathrm{G}$. The periaqueductal gray controls brainstem emotional motor systems including respiration. Prog Brain Res. 2014;209:379-405. doi:10.1016/B978-0-444-63274-6.00020-5

37. Craig AD. How do you feel? interoception: the sense of the physiological condition of the body. Nat Rev Neurosci. 2002;3 (8):655-666.

38. Critchley HD, Mathias CJ, Dolan RJ. Neuroanatomical basis for firstand second-order representations of bodily states. Nat Neurosci. 2001;4(2):207-212.

39. Saper CB. The central autonomic nervous system: conscious visceral perception and autonomic pattern generation. Annu Rev Neurosci. 2002;25:433-469.

40. Berthoud HR, Neuhuber WL. Functional and chemical anatomy of the afferent vagal system. Auton Neurosci. 2000;85(1-3):1-17.

41. Klarer M, Arnold M, Günther L, Winter C, Langhans W, Meyer U. Gut vagal afferents differentially modulate innate anxiety and learned fear. J Neurosci. 2014;34(21):7067-7076. doi:10.1523/ JNEUROSCI.0252-14.2014 
42. Klarer M, Krieger JP, Richetto J, et al. Abdominal vagal afferents modulate the brain transcriptome and behaviors relevant to schizophrenia. J Neurosci. 2018;38:1634-1647. doi:10.1523/ JNEUROSCI.0813-17.2017

43. Foreman RD, Qin C, Jou CJ. Spinothalamic system and viscerosomatic motor reflexes: functional organization of cardiac and somatic input. In: King HH, Jänig W, Patterson MM, eds. The Science and Clinical Application of Manual Therapy. Edinburgh, Scotland: Churchill Livingstone Elsevier; 2011:11-127.

44. Jänig W, Green P. Acute inflammation in the joint: its control by the sympathetic nervous system and neuroendocrine systems. Auton Neurosci. 2014;182:42-54. doi:10.1016/j.autneu.2014.01.001

45. Taylor AG, Goehler LE, Galper DI, Innes KE, Bourguignon C. Top-down and bottom-up mechanisms in mind-body medicine: development of an integrative framework for psychophysiological research. Explore (NY). 2010;6(1):29-41. doi:10.1016/j explore.2009.10.004

46. Janig W. Neurobiology of visceral afferent neurons: neuroanatomy, functions, organ regulations and sensations. Biol Psychol. 1996;42 (1-2):29-51. doi:10.1016/0301-0511(95)05145-7

47. Verberne AJ, Saita M, Sartor DM. Chemical stimulation of vagal afferent neurons and sympathetic vasomotor tone. Brain Res Brain Res Rev. 2003;41(2-3):288-305. doi:10.1016/s0165-0173(02)00269-2

48. Duncan BL, Moynihan DW. Applying outcome research: intentional utilization of the client's frame of reference. Psychother Theory Res Pract Train. 1994;31(2):294.

49. Lambert MJ, Simon W. The therapeutic relationship: central and essential in psychotherapy outcome. In: Hick SF, Bien T, eds. Mindfulness and the Therapeutic Relationship. New York, NY: Guilford Press; 2008:19-33.

50. Martin DJ, Garske JP, Davis MK. Relation of the therapeutic alliance with outcome and other variables: a meta-analytic review. J Consult Clin Psychol. 2000;68(3):438.
51. Geller SM, Greenberg LS. Therapeutic Presence: A Mindful Approach to Effective Therapy. Washington, DC: American Psychological Association; 2012

52. Siegel DJ. Mindsight: The New Science of Personal Transformation New York, NY: Bantam Books; 2010.

53. Geller SM, Porges SW. Therapeutic presence: neurophysiological mechanisms mediating feeling safe in therapeutic relationships. J Psychother Integrat. 2014;24(3):178.

54. Liem T. Kraniosakrale Osteopathie. 7th ed. Stuttgart, Germany: Thieme; 2018

55. Porges SW, Liem T. Die polyvagaltheorie in der osteopathie. Osteopathische Medizin. 2016;17(2):14-20. doi:10.1016/S1615-9071 (16)30012-0

56. Elkiss ML, Jerome JA. Touch—more than a basic science. J Am Osteopath Assoc. 2012;112(8):514-517.

57. Liem TVan den Heede P., Morphodynamics in Osteopathy. An Integrative Approach to Cranium, Nervous System and Emotions. Pencaitland, Scotland: Handspring Publishing; 2016.

58. Liem T. Morphodynamik in der Osteopathie. 2nd ed. Stuttgart, Germany: Haug; 2013.

59. Liem T. Wechselseitige beziehungsdynamiken und subjektive ansätze in der osteopathie. Osteopathische Medizin. 2011;12(2):4-7.

60. Liem T. Osteopathie und (Hatha-)yoga. Osteopathische Medizin. 2009;10(1):21-27

61. Fennig GA, Shubrook JH. Inpatient osteopathic structural examinations: is "red tape" getting in the way of personalized patient care? J Am Osteopath Assoc. 2008;108 (7):327-332.

62. What is brainspotting? Brainspotting website. https://brainspotting.com/ about-bsp/what-is-brainspotting/. Accessed February 1, 2019.

(C) 2020 American Osteopathic Association 